Mansoura University

Faculty of Education

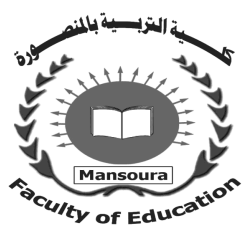

PROGRAMME PROPOSÉ BASÉ SUR LA RÉTROACTION POUR LE DÉVELOPPEMENT DES COMPÉTENCES LANGAGIÈRES PRAGMATIQUES EN FLE CHEZ LES ÉTUDIANTS DU CYCLE SECONDAIRE

\author{
By \\ Mohamed Salah ABDELAAL \\ Doctorat en pédagogie (Didactique du FLE)
}

Journal Of The Faculty Of Education-Mansoura University

No. 110 - April. 2020 


\title{
Programme proposé basé sur la rétroaction pour le développement des compétences langagières pragmatiques en FLE chez les étudiants du cycle secondaire
}

\author{
Mohamed Salah ABDELAAL \\ Doctorat en pédagogie (Didactique du FLE)
}

\section{Résumé de la recherche}

La recherche actuelle a pour but de développer quelques compétences langagières pragmatiques en FLE chez les étudiants de la deuxième année du cycle secondaire à travers l'emploi d'un programme proposé basé sur la rétroaction en examinant son effet. L'échantillon de cette recherche se compose de 30 étudiants parmi ceux de la deuxième année secondaire constituant le groupe expérimental de cette recherche. L'outil de la présente recherche porte sur un test des compétences langagières pragmatiques en FLE.

Après avoir enseigné le programme proposé basé sur la rétroaction, les résultats ont conduit à la vérification de l'hypothèse de la recherche:

"Il y a des différences significatives statistiquement au niveau $P \leq 0,05$ entre les moyennes des notes d'étudiants de l'échantillon de la recherche aux prélposttests des compétences langagières pragmatiques en faveur du post test»

\section{Introduction}

L'apprentissage d'une langue, comme tout apprentissage, est un processus, personnel et social, qui se manifeste à travers la responsabilisation de l'apprenant face à la construction de ses savoirs et à leur utilisation dans des contextes de plus en plus variés et complexes. L'enseignement/apprentissage du français langue étrangère a pour objectif de développer, chez les apprenants, des compétences de communication à l'oral (écouter/parler) et à l'écrit (lire/écrire). Ils seront amenés, progressivement, à communiquer à l'oral comme à l'écrit dans des situations scolaires adaptées à leur niveau cognitif. En effet, il s'agit non seulement d'enseigner le français mais aussi d'enseigner comment communiquer en cette langue en adoptant des méthodes spécifiques par le biais de certains outils. (Alrabadi, 2011: 15-34)

La compétence pragmatique est l'une des compétences communicatives qu'on doit maîtriser pour pouvoir se communiquer. Elle traite de la connaissance que l'apprenant a des principes selon lesquels les messages sont:

- organisés, structurés et adaptés (compétence discursive). 
- utilisés pour la réalisation de fonctions langagières (compétence fonctionnelle).

- segmentés selon des schémas interactionnels et transactionnels (compétence de conception schématique). (Conseil de l'Europe, 2001: 96-101)

Ainsi, la compétence pragmatique recouvre l'utilisation fonctionnelle des ressources de la langue (réalisation de fonctions langagières, d'actes de parole,...etc) en s'appuyant sur l'interaction entre un locuteur et un interlocuteur. Le locuteur (émetteur) utilise un système de codes linguistiques et sociaux, pour produire un message destiné à un interlocuteur (récepteur). Dans ce sens, la pragmatique désigne l'étude sociale, cognitive, et culturelle du langage et de la compétence communicative qui contient la compétence grammaticale, la compétence socioculturelle et la compétence stratégique. Ces compétences contiennent les traits des compétences langagières parce que les composants prennent en considération l'utilisation de la langue. (Viljamaa, 2012: 16-17)

Ajoutons que la compétence pragmatique se compose du savoir de la langue et également du savoir de l'emploi de la langue. L'apprenant mobilise des aptitudes générales pour réaliser des intentions langagières. Ces aptitudes générales prennent la forme de savoirs différents (savoir, savoirfaire, savoir-être) qui s'associe à une compétence communicative plus spécifiquement linguistique. Selon le CECR, trois composantes caractérisent cette compétence communicative: les compétences linguistiques, les compétences sociolinguistiques et les compétences langagières. (Noyon, 2011: 273)

Si un apprenant n'ose pas relever les erreurs grammaticales, lexicales et pragmatiques, il se peut donc que l'enseignant soit le seul à pouvoir corriger l'apprenant. Il faut donner à l'apprenant les outils pour qu'il puisse s'auto évaluer et d'une façon plus autonome. (Martinot, 2006)

Il est nécessaire de développer certaines compétences langagières pragmatiques en FLE comme à titre d'exemple: utiliser la compétence fonctionnelle. Cette compétence comprend quelques sous-compétences comme: savoir quelles formes linguistiques expriment quelles fonctions particulières, définir l'utilisation fonctionnelle d'énoncés simples (courts), construire des phrases correctes grammaticalement et orthographiquement, donner et demander des informations, exprimer des attitudes (accord/désaccord, permission, préférences, goût, excuses), faire suggérer (conseils, demande d'aide), établir des relations sociales (s'adresser aux gens, saluer, présenter, prendre congé), remédier à la communication écrite 
(déscription, commentaire, explication), recevoir de la rétroaction, reconnître ouvertement ses erreurs, avoir confiance en soi. De plus, l'apprenant doit être capable de raisonner sur la langue qu'il utilise et employer ses connaissances linguistiques en communication écrite sous l'aspect du niveau pragmatique.

Dans ce sens, il faut mettre l'accent sur la nécessité de l'utilisation de nouvelles méthodes visant à développer ces compétences langagières pragmatiques en FLE et transmettant le plaisir d'apprendre grâce à une démarche riche en activités variées et motivantes. Parmi ces méthodes celles de la rétroaction qui permettent aux enseignants et aux étudiants de contrôler leurs actions dans une situation donnée. Au sein de cette méthode, l'étudiant va construire de nouvelles connaissances à partir des ressources mises en place par l'enseignant qui observe, à son tour leurs phrases erronnées. Par conséquent, les théories d'apprentissage s'entendent pour dire que la rétroaction joue un rôle important dans les classes du français langue étrangère, surtout à l'écrit.

La notion de la rétroaction renvoie d'une part à l'évaluation formative qui sert à vérifier le niveau de compétence d'acquisition des connaissances de l'étudiant. Cette sorte d'évaluation semble devenir un des éléments central à l'apprentissage puisqu'elle permet de rassurer les étudiants sur ce qu'ils entreprennent et de les encourager à prendre des risques et accepter leurs erreurs. Un progrès de la confiance en soi des étudiants dans leurs capacités scolaires comptent parmi les effets positifs observés de l'évaluation formative. De ce fait, la rétroaction favorise les apprentissages des étudiants, et a également un grand impact sur la confiance en soi des étudiants. Elle permet de se souligner les points forts et les points faibles des apprenants et les amène par conséquent à développer leur autonomie d'apprentissage.

En somme, à travers la rétroaction, l'enseignant peut également récolter des informations sur la progression de ses étudiants (El Shirbini, 2015: 24-35).

En fait, Il y a un lien entre les types d'erreurs et les stratégiede la rétroaction, l'étudiant apprend à partir de ses erreurs. Enfin, on a besoin de classes qui cultivent le courage de l'erreur considérée comme une partie essentielle de l'apprentissage. Le type d'erreurs influence le choix de la stratégie de la rétroaction (Salama, 2010: 1-8).

Les enseignants de français utilisent la rétroaction afin de rétroagir sur l'aspect pragmatique de la production écrite. Elle vise également à explorer si la révision des étudiants varient selon la catégorie de l'erreur 
(grammaticale, lexicale, pragmatique). La correction des produits écrits des apprenants du cycle secondaire devrait porter sur les erreurs qui nuisent ou empêchent la communication. Donc, si les apprenants produisent des erreurs à l'écrit, l'enseignant peut intervenir, au moyen de rétroactions correctives écrites (directes et déductives), pour les aider à s'améliorer.

Rappelons que le fait qu'il y a un lien entre la rétroaction et les composantes grammaticales et lexicales sur lesquelles portent cette rétroaction qui met en évidence la communication à l'écrit. À la lumière de celà, nous pensons que les rétroactions correctives écrites de l'enseignant aident à acquérir les formes correctes de la langue française, donc, la production ècrite dans la langue étrangère (ici le français) et favorise son acquisition (Asselin, 2006: 8).

Donner aux étudiants une rétroaction sur leurs progrès les aide à rester confiants et leur permet de se corriger si besoin. Mettre en place des objectifs personnels nécessite de connaître le niveau de l'étudiant et de spécifier où vous voulez qu'il parvienne en temps voulu. L'accent sur les progrès personnels permet aux étudiants d'entrer en compétition avec eux même en réussissant de leur mieux. En conclusion, quand les enseignants cherchent, ou au moins sont attentifs, la rétroaction des étudiants concernant ce qu'ils savent, leur de degré de production écrite, leurs erreurs, leur manque de confiance en soi, alors l'enseignant et l'apprentissage peuvent être synchronisés et puissants (Hattie, 2007: 81-112).

\section{Sensibilisation au problème}

La sensibilisation au problème de la recherche actuelle consiste aux résultats des études antérieurs consultées, l'expérience personnelle du chercheur et l'étude pilote qu'il a effectuée comme le suivant:

\section{Les études antérieures}

Les résultats des études antérieures consultées comme: Mahmoud (2003), Fabienne (2004), Maraheel (2004), Dalmasoo (2009), Viljamaa (2012), Tognet (2014). Toutes ces études ont montré que le niveau des apprenants est faible en ce qui concerne les compétences langagières pragmatiques en FLE dans le domaine des langues en général et des langues étrangères en particulier.

\section{L'expérience personnelle du chercheur}

Ces résultats se sont mis en d'accord avec l'expérience personnelle du chercheur, en tant que premier enseignant de français au secteur gouvernemental, sur l'existence d'une faiblesse chez les étudiants du cycle secondaire des compétences langagières pragmatiques en FLE en comettant des errreurs grammaticales, lexicales et pragmatiques. 


\section{L'étude pilote}

En vue de valider ces observations, le chercheur a effectué une étude pilote comme le suivant:

Tout d'abord, il a élaboré un test concernant certaines compétences langagières pragmatiques en FLE en l'appliquant ensuite sur un échantillon d'étudiants en 2ème année secondaire (15 étudiants) pour évaluer le degré de leur usage/emploi de ces compétences langagières pragmatiques en FLE. Les résultats de cette étude pilote ont montré que le niveau des étudiants est faible en ce qui concerne ces compétences. Ces résultats sont indiqués dans le tableau suivant:

\section{Tableau (1)}

Résultats de l'étude pilote

\begin{tabular}{|c|c|c|}
\hline Compétences langagières pragmatiques & $\begin{array}{c}\text { No } \\
\text { des } \\
\text { étudiants }\end{array}$ & $\begin{array}{c}\text { \% des } \\
\text { réponses } \\
\text { correctes }\end{array}$ \\
\hline 1- Savoir quelles formes linguistiques doit-il utiliser. & 15 & $47 \%$ \\
\hline $\begin{array}{l}\text { 2- Construire des phrases correctes (lexicales, grammaticales } \\
\text { et pragmatiques). }\end{array}$ & 15 & $45 \%$ \\
\hline $\begin{array}{l}\text { 3- Etablir des relations sociales (s'adresser aux gens, saluer, } \\
\text { présenter, prendre congé). }\end{array}$ & 15 & $40 \%$ \\
\hline $\begin{array}{l}\text { 4- } \begin{array}{c}\text { Remédier les erreurs à la communication écrite } \\
\text { (déscription, commentaire, explication) }\end{array} \\
\end{array}$ & 15 & $33 \%$ \\
\hline $\begin{array}{l}\text { 5- Exprimer des attitudes (accord/désaccord, permission, } \\
\text { préférences, goût, excuses) }\end{array}$ & 15 & $27 \%$ \\
\hline
\end{tabular}

Les résultats indiqués dans le tableau no. (1) mettent en relief l'existence d'une faiblesse par rapport à l'usage/emploi des compétences langagières pragmatiques en FLE chez les étudiants de la $2^{\text {ème }}$ année du cycle secondaire. Ainsi, pour déterminer les types d'erreurs, le chercheur a demandé de l'échantillon de cette étude pilote de rédiger trois paragraphes en cinq lignes de/d' :

- Engager une conversation avec un ami sur la profession de ton père en utilisant ces mots (la profession - la nature du travail- le lieu du travail).

- Décrire l'état de temps en hiver : (pleuvoir- il y a-le temps- nuages)

- Ecrire 4 phrases sur le projet de ta fammille pour le week-end prochain en utilisant ces mots (cinéma, restaurant, aller, regarder).

La correction des copies du corpus a montré que les membres de l'échantillon ont commis tant d'erreurs lexicales, grammaticales et pragmatiques. Les résultats de cette correction sont montrés dans le tableau suivant: 
Tableau (2)

Types d'erreurs commises

\begin{tabular}{|l|c|c|}
\hline \multicolumn{1}{|c|}{ Types d'erreurs } & $\begin{array}{c}\text { No } \\
\text { d'erreurs }\end{array}$ & Pourcentage \\
\hline 1. Grammaticales & 202 & 35.56 \\
\hline 2. Lexicales & 159 & 27.99 \\
\hline 3. pragmatiques & 207 & 36.44 \\
\hline
\end{tabular}

Les résultats dans le tableau no. (2) montrent qu'il y a réellement un problème et l'existence de tant d'erreurs qui considère comme une partie intégrante du contenu et de la forme du texte écrit, pour cela, il faut apprendre à en tolérer un certain nombre et ne plus faire trop de commentaires ou de corrections avec le stylo rouge.

\section{Problématique de la recherche}

Le problème de cette recherche réside dans la faiblesse du niveau des étudiants de la deuxième année secondaire en ce qui concerne les compétences langagières pragmatiques en FLE ce qui demande de répondre à la question principale suivante: "Quelle est l'impact d'un programme proposé basé sur la rétroaction pour le développement des compétences langagières pragmatiques en FLE chez les étudiants du cycle secondaire?"

D'aprés cette question, on pose quelques questions:

- Quelles sont les compétences langagières pragmatiques en FLE nécessaires aux étudiants de la deuxième année secondaire?

- Jusqu'à quel point les étudiants de la deuxième année secondaire maîtrisent-ils les compétences langagières pragmatiques en FLE?

- Quelles sont les caractéristiques d'un programme proposé basé sur la rétroaction visant le développement des compétences langagières pragmatiques en FLE chez les étudiants de la deuxième année secondaire?

- Quel est l'impact d'un programme proposé basé sur la rétroaction visant le développement des compétences langagières pragmatiques en FLE chez les étudiants de la deuxième année secondaire?

\section{Hypothèse de la recherche}

La présente recherche essaye de vérifier l'hypothèse suivante:

Il y a des différences significatives statistiquement au niveau de $\mathrm{P} \leq 0.05$ entre les moyennes des notes d'étudiants de l'échantillon de la recherche aux pré/post-tests des compétences langagières pragmatiques en FLE en faveur du post-test 
5. Objectifs de la recherche

Cette étude vise à réaliser les objectifs suivants

- Développer quelques compétences langagières pragmatiques en FLE chez les étudiants de la deuxième année secondaire et la confiance en soi.

- Evaluer le programme proposé basé sur la rétroaction pour le but de développer la performance des étudiants de la deuxième année secondaire en ce qui concerne des compétences langagières pragmatiques en FLE.

\section{Délimites de la recherche}

\section{L'étude actuelle se limitera à :}

- Un échantillon des étudiants de la deuxième année secondaire dans une école secondaire à Mansourah.

- Quelques compétences langagières pragmatiques en FLE.

- Les deux unités (8 leçons) (le programme proposé basé sur la rétroaction) pour développer les compétences langagières pragmatiques en FLE chez les étudiants de la deuxième année secondaire.

\section{Echantillon de la recherche}

L'échantillon de cette recherche se compose d'un seul groupe de (30) étudiants parmi ceux de la 2ème année secondaire. Ce choix est justifié par le fait que ces étudiants ont déjà acquis un bagage linguistique suffisant en première année secondaire. Ce qui leur permet de développer leurs compétences langagières pragmatiques en FLE.

\section{Méthodologie de la recherche}

La recherche actuelle utilise à la fois la méthode quasi-expérimentale et la méthode descriptive analytique comme suit:

- La méthode descriptive analytique pour passer en revue les études antérieures portant sur la rétroaction et déterminer les compétences langagières pragmatiques en FLE chez les étudiants de la deuxième année secondaire.

- La méthode quasi-expérimentale pour mesurer l'impact du programme proposé basé sur la rétroaction pour le développement de quelques compétences langagières pragmatiques en FLE chez les étudiants de la deuxième année secondaire.

\section{Outil et le matériel de la recherche}

- Une grille des compétences langagières pragmatiques en FLE nécessaires aux étudiants de la deuxième année secondaire. 
- Un pré/post test des compétences langagières pragmatiques en FLE chez les étudiants de la $2^{\text {ème }}$ année secondaire.

- Un programme proposé basé sur la rétroaction.

\section{Procédures de la recherche}

Pour répondre aux questions de l'étude. L'étude suivra les étapes suivantes:

1. Passer en revue les études antérieures et les écrits théoriques portant sur:

- Les méthodes de l'enseignement/apprentissage du FLE surtout ceux de la rétroaction.

- Les compétences langagières pragmatiques en FLE.

2. Elaborer une grille des compétences langagières pragmatiques en FLE nécessaires aux étudiants de la deuxième année du cycle secondaire.

3. Elaborer un test pour mesurer le niveau des étudiants de la deuxième année du cycle secondaire par rapport aux compétences de la grille élaborée.

4. Élaborer le matériel de la recherche qui est un programme proposé basé sur la rétroaction comportant un cahier d'activités (Cahier d'étudiant) et un guide pédagogique (Guide de l'enseignant) et visant le développement des compétences langagières pragmatiques en FLE chez les étudiants du cycle secondaire

5. Soumettre les outils et le matériel de la recherche au jury composé des spécialistes et experts pour vérifier leur validité apparente.

6. Modifier les outils et le matériel de la recherche selon les points de vue du jury.

7. Choisir l'échantillon de la recherche qui est composé de (30) étudiants parmi ceux de la 2ème année secondaire.

8. Pré-appliquer l'outil de la recherche: le test des compétences langagières pragmatiques en FLE.

9. Enseigner le programme proposé basé sur la rétroaction.

10. Post-appliquer l'outil de la recherche: le test des compétences langagières pragmatiques en FLE.

11. Analyser et interpréter les résultats de la recherche.

12. Présenter les recommandations et les suggestions de la recherche.

11. Terminologies de la recherche

On va exposer les terminologies de cette recherche comme suit:

\section{La rétroaction}

Elle est « un des moyens, dont dispose l'ensegnant pour aider l'apprenant à réaliser un apprentissage, est de lui fournir une information 
portant sur l'adéquation ou l'indéquation de sa réponse par rapport à une performance attendue» (Doré, 1981). Lighbown et Spada (2006) parlent qu'il s'agit de toute indication qui montre aux apprenants que leur production n'est pas correcte. Quant à Mory (1996) la rétroaction est définie comme étant n'importe quelle réaction qui informe l'apprenant que sa réponse n'est pas la bonne ou la réponse désirée (Hassan, 2011: 42-43).

Ainsi, la rétroaction est généralement définie comme toute correction explicite ou implicite, métalinguistique ou incitation de la part de l'enseignant indiquant que la production écrite de l'apprenant est erronée (Salama, 2010: 3-8).

À partir de ces définitions, la rétroaction se définit opérationnellement dans cette recherche comme, la manière d' améliorer l'apprentissage des étudiants et créer un climat propice à l'apprentissage dans la salle de classe. Elle renseigne les étudiants sur leur apprentissage et leur fournit des renseignements au sujet de leurs forces, des améliorations requises et de la façon dont elles et ils peuvent s'améliorer. Elle est aussi un complétement de l'évaluation qui permet de préciser ce qui a été bien fait et ce qui doit être amélioré).

\section{Les compétences langagières pragmatiques en FLE}

Pour le CECR (2000: 96-101), la pragmatique signifie l'utilisation fonctionnelle des ressources de la langue (réalisation de fonction langagières, d'actes de parole), elle a ainsi pour objectif de réinsérer l'énoncé produit dans l'acte de communication, c'est- à-dire de décrire les relations entre l'énoncé, les interlocuteurs et la situation de communication.

Alors que la compétence langagière pragmatique est la compétence à communiquer à l'écrit. La pragmatique et la communication sont étroitement liées et, en fait, la compétence pragmatique est souvent considérée comme une composante de la compétence de communication.

De cela, la définition opérationnelle de la compétence langagière pragmatique dans cette recherche est la capacité à utiliser l'écriture pour exprimer les idées ou des informations dans la vie quotidienne de tous les jours. Au sein de la compétence pragmatique, nous étudions la capacité des étudiants de la langue française de produire des actes de langage qui représentent une partie importante de la pragmatique. C'est pourquoi, nous avons choisi d'examiner ces actes à travers l'emploi de la stratégie de rétroaction.

\section{Cadre théorique}

Le cadre théorique de cette recherche comporte deux volets principaux: les compétences langagières pragmatiques en FLE et la stratégie de la rétroaction. 


\subsection{Les compétences langagières pragmatiques en FLE}

On va exposer Conception des compétences langagières pragmatiques, le lien entre les Compétences langagières pragmatiques et la communication. Evaluation des compétences langagières pragmatiques en FLE, définition de la rétroaction, ....etc.

\subsubsection{Conception des compétences langagières pragmatiques en FLE}

Il est vrai que les compétences langagières et langagières écrites en FLE se composent du savoir de la langue et également l'emploi du savoir afin de réaliser la communication à travers la diversité des situations langagières. L'apprenant qui joue un rôle comme un acteur social, il mobilise des aptitudes générales pour réaliser des intentions langagières. Ces aptitudes générales prennent la forme de différents savoirs (savoir, savoir-faire, savoir-être) qui s'associe à une compétence communicative plus spécifiquement linguistique (Noyon, 2011: 273).

D'après le CECRL (2001), la cométence communicative langagière est composée de trois sous compétences suivantes:

- Les compétences linguistiques

Il s'agit des savoirs et savoirs-faire relatifs au lexique, à la grammaire, à la sémantique et à l'orthographe.

- La compétence sociolinguistique

Elle renvoie aux parmètres socioculturels de l'utilisation de la langue. Sensible aux normes sociales (règles de politesse, régulation entre générations).

- La compétence pragmatique

Elle recouvre l'utilisation fonctionnelle des ressources de la langue (réalisation de fonctions langagières, d'actes de parole). Elle renvoie également à la maîtrise du discours écrit à sa cohésion et cohérence. La compétence pragmatique correspond globalement à la capacité de bien gérer les interactions de la vie quotidienne.

Dès lors, la pragmatique attire notre attention sous la compétence pragmatique qui signifie l'usage social du langage. Dans cette perspective, nous signalons que communiquer c'est utiliser des règles linguistiques ou un code linguistique (compétence linguistique) rapportées à une action (compétence pragmatique) dans un contexte socioculturel (compétence sociolinguistique).

A la lumière de ce qui pécède, la compétence pragmatique est une des compétences communicatives qu'on doit maîtriser pour se communiquer par écrit. 
Dans ce sens, l'aspect pragmatique de la production écrite se définit comme une tache complexe qui peut être envisagée sous différents aspects (Zesiger, 1995: 54) cité par Nassar (2017: 16):

- Communicationnelle: le fait de s'inscrire dans une démarche de communiquer en choississant de recourir a l'écrit;

- Sémantique: le sens du message de l'écrit;

- Linguistique: les mots, les termes utilisés, leur organisation;

- Socio-culturel: les règles et les normes de communication institutionnellement inscrites.

- pragmatique: les connaissances sur les intentions de communication.

\subsubsection{Le lien entre les Compétences langagières pragmatiques en FLE} et la communication

Dès le début, écrire devient un acte de communication, un savoir et un savoir-faire spécifique permettant à l'apprenant de s'exprimer et de communiquer dans la vie courante. En effet, l'approche communicative et actionnelle du CECRL a mis l'accent sur l'aspect pragmatique de la production écrite qui est considéré les études pratiques langagières d'une langue d'apprentissage (Desmon, 2005: 45).

Certainement, quand nous demandons aux apprenants de rédiger des textes simples et courts autour de ce qui impliquent dans leur vie de tous les jours, c'est l'aspect pragmatique de la production écrite. Par exemple, dans l'activité de production écrite au niveau pragmatique, nous mettons l'apprenant dans une situation qui décrit une réalité dans la vie celle l'état du climat pendant la saison. Nous lui demandons de faire un travail d'écriture qui consiste à tenir le discours écrit dans un dialogue (Khaled, 2010: 10).

Quant à la communication n'étant plus uniquement réservée à l'oral mais également à l'écrit. Chaque langue vivante se parle et s'écrit. En d'autre terme, la langue parlée et langue écrite correspondent à de différentes situations de communication. C'est-à-dire, il y a un discours oral et un discours écrit. Dans notre recherche, nous nous intéressons à l'aspect pragmatique de la production écrite au niveau A1 du DELF pour aider les apprenants à produire un petit texte correspondant à la situation communicationnelle en utilisant les mots donnés ou un lexique approprié au sujet traité, par exemple: Décris l'état de temps en hiver : (pleuvoir- il y a le temps- nuages ). Au point précédent, pourquoi l'enseignement de cet aspect est nécessaire parce que les apprenants ne sont pas capables de s'exprimer par écrit ce qu'ils savent déjà et ils n'ont pas assez d'occasions pour apprendre à communiquer. 
De ce fait, grâce à l'utilisation de l'aspect pragmatique de la production écrite, l'apprenant sera capable de s'exprimer en situation réelle de communication ses souhaits, donner et demander une information par écrit en construisant des phrases simples et correctes, pour cela, nous proposons des activités d'entrainement qui l'aident à utiliser certaines compétences (ici les compétences langagières en FLE).

De plus, l'aspect pragmatique de la production écrite est une activité langagière à part entière. Cet aspect se traduit par la capacité à pratiquer la langue dans de diverses activités d'entrainement: raconter, décrire, expliquer, ....etc (Biscons, 2008) cité par (Esmat, 2016: 21).

Il est évident que tout apprentissage et toute utilisation d'une langue se passent dans une situation par laquelle tout acte de communication a un but à réaliser: demander et donner des informations, offrir, refuser une chose, exprimer une idée, donner une opinion, soulignons que la situation de communication de façon écrite (ici l'aspect pragmatique de la production écrite) est l'utilisation de la langue dans la vie sociale. En bref, l'écriture est une activité sociale fondamentale, savoir s'exprimer par écrit fait partie des compétences (ici les compétences langagières en FLE) à acquérir par chacun en mettant l'apprenant en situation d'écriture pendant un moment d'apprentissage/enseignement de la langue étrangère.

\subsubsection{Evaluation des compétences langagières pragmatiques en FLE}

Premièrement, l'évaluation de la compétence pragmatique peut permettre d'apprécier la capacité communicative d'un apprenant en contexte.

Au sein de la compétence pragmatique, nous allons évaluer la capacité des apprenants de la langue française de produire des actes de parole qui représentent une partie importante de la compétence pragmatique. La pragmatique étudie le langage en situation et elle est l'étude du langage en acte. C'est pourquoi, nous évaluons les compétences langagières chez les étudiants à travers un test de compétence pragmatique qui contient quelques situations et nous leur demandons d'écrire ce qu'ils diront dans ces situations. Avant ce test, nous entraine les apprenant à utiliser la langue française dans la vie quotidienne en leur présentant des activités d'entrainement.

D'après Kasper (1997: 10) et Neuvonen (2015: 33-68), il existe un grand nombre d'activités d'entrainement en permettant de développer les actes de parole puis d'évaluer les performances des compétences langagières dans de différentes/nouvelles situation de communication:

- les activités discursives: La plupart des exercices dans ces catégories permettent aux étudiants d'améliorer leur capacité de 
structurer les textes en termes d'organisation thématique, de cohérence et de cohésion. Ces exercices également devraient inclure d'autres informations langagières en ce qui concerne les conventions liées à la rédaction de certains types de textes. Les types de ces activités discursives écrites sont:

○ Écrire un dialogue;

- Écrire un message;

○ Écrire une lettre;

○ Écrire un courriel;

En somme, ces activités ci-dessus contiennent des situations de communication et la tâche de l'apprenant est d'écrire ces types. Les activités discursives de la catégorie «écrire un dialogue», par ex, nous demandons aux étudiants d'écrire un dialogue qui se passe au magasin de vêtements et l'autre dialogue imaginaire par exemple est entre l'étudiant et son ami français, sur un monument historique en Égypte,...etc. Les activités discursives de la catégorie «écrire un message» incluent une situation de communication similaire: il s'agit d'écrire un message de vacances et de décrire où et avec qui on est et ce qu'on va faire pendant les vacances. Il y a également des activités discursives comme : écrire un mail à un copain ou un courriel à un correspondant sur le chat (sur Facebook). D'après les activités discursives écrites, les apprenants doivent apprendre à adapter les textes aux contextes et situations de la communication et prendre aussi en compte le niveau de la politesse.

- les activités fonctionnelles: dans le CECRL, types de ces activités incluent des fonctions très simples, comme: les remerciements et dire au revoir, qui peuvent être complétées avec un seul mot «Salut», «Au revoir» «Merci», la description, exprimer son opinion, exprimer ses goûts et ses préférences (aimer/ne pas aimer quelque chose), se présenter et exprimer l'obligation). Par ex, nous demandons aux apprenants d'écrire des descriptions liées aux sujets traités en donnant leur opinion sur des choses.

Les activités schématiques d'interaction sociale: dans le CECR, l'activité schématique est liée de près à l'activité fonctionnelle. Types de ces activités d'entrainement comme: proposer et refuser un rendez-vous, parler de son état de santé, demander son chemin et l'achat de marchandises.

- les activités sociolinguistiques, la compétence sociolinguistique n'est pas une composante explicite de la compétence pragmatique dans le CECRL, mais une composante indépendante de la 
compétence de communication. Cette compétence est considérée comme un élément primordial de la compétence pragmatique. Types de ces activités d'entrainement sont: marqueurs de relations sociales qui incluent l'usage et le choix des salutations dans un exercice, et l'usage et le choix des formes d'adresse dans l'autre «Madame», «Monsieur», et «Mademoiselle». La catégorie des règles de politesse qui inclut des exercices liés à la manière de faire des compliments en français dans deux exercices consécutifs, et le vouvoiement est abordé dans deux exercices.

Dans ce qui vient de ces paragraphes précédents, dans les productions écrites au niveau pragmatique (ici les activités d'entrainement), un ou plusieurs actes de parole sont demandés aux apprenants et l'on peut donc évaluer la réalisation de ces actes. Il s'agit ici des compétences langagières en FLE à évaluer pour des apprenants qui doivent être cabaple de décrire ou présenter simplement des gens, des activités quotidiennes, ce qu'on aime ou pas en classe.

Sans doute, l'évaluation est un moyen de voir comment les étudiants progressent. Il important de citer que l'évaluation n'est pas le role des enseignants. Les apprenants, eux aussi, profitent de l'analyse de leur texte pour détecter leurs difficultés et tanter de développer des stratégielangagières des différentes formes de l'activité d'entrainement communicative. Ces stratégiesont considérées comme le moyen utilisé par l'usage d'une langue afin de répondre aux exigences de la communication en situation pour améliorer les productions écrites des apprenants au niveau pragmatique.

Finalement, d'après le Cadre européen commun de référence des langue vivantes CECRL (2001: 164-169) présente des critères pour les descripteurs des niveaux communs de références pour le niveau A1 concernant l'évaluation de l'aspect pragmatique de la production écrite comme le suivant: les apprenants de ce niveau (ici les étudiants du cycle secondaire) peuvent écrire une carte postale simple, envoyer des nouvelles de vacances, remplir des formulaires demandant des renseignements personnels, décrire des objets comme un lieu et un logement ou des personnes.

Pour en finir, dans notre recherche pour développer les compétences langagières pragmatiques en FLE, on va utiliser un programme proposé basé sur la rétroaction. 


\subsection{La rétroaction}

On va exposer la définion et l'importance de la rétroaction, ses types et les types d'erreurs et les techniques de la rétroaction.

\subsubsection{Définition et imortance de la rétroaction}

On peut définir la rétroaction simplement comme un ensemble de réactions à des énoncés d'apprenants comportant une erreur. La rétroaction en enseignement désigne habituellement l'information qui est donnée par un-e enseignant-e à un-e étudiant-e suite à l'évaluation d'un travail écrit ou oral. La rétroaction n'est pas seulement un outil pour les étudiant-e-s, mais peut également être utile pour l'enseignant-e. À travers La rétroaction, l'enseignant-e peut également récolter des informations sur leur progression et identifier des difficultés éventuelles avec un sujet particulier. Il est alors possible de cibler les points qui devront être repris par la suite.

La rétroaction peut être oral, écrit ou visuel. Le type de feedback dépend du caractère des activités pédagogiques. Ainsi, les professeurs peuvent expliquer aux apprenants leurs erreurs et les apprenants ont des possibilités de poser des questions supplémentaires. En général, ce sont les enseignants qui choisissent le type de rétroaction selon la situation et les activités langagières. Ils peuvent le donner individuellement ou bien pour toute la classe lorsque beaucoup d'apprenants font les mêmes erreurs. (Pardu, 2015: 23-24)

La rétroaction est un complément de l'évaluation qui permet de préciser ce qui a été bien fait et ce qui doit être amélioré. Peut aussi être faite en dehors du contexte des évaluations; constitue une forme de reconnaissance du travail de l'étudiant. Évaluer, ce n'est pas seulement évaluer le contenu. C'est aussi évaluer le processus d'apprentissage. Lorsqu'un projet est un processus, on fait des commentaires pendant le processus. À la fin, il n'y a pas de rétroaction.

Bien utilisées, les rétroactions favorisent les apprentissages de l'étudiant. Elles ont également un grand impact sur l'estime de soi des étudiants. Elles permettent de souligner les forces de l'étudiant dans ses apprentissages. Bien guidés, tous les étudiants peuvent arriver à vaincre leurs erreurs et à relever les défis de l'atteinte des objectifs. Lorsque l'enseignant identifie avec précision les forces et les défis de l'étudiant, il lui permet de voir le chemin à parcourir et les points sur lesquels il doit concentrer son énergie.

Le but de la rétroaction est de diminuer l'écart entre le point de départ des élèves et l'endroit où vous voulez les conduire. La rétroaction diminue 
cet écart en permettant à vos élèves de savoir comment ils s'en sortent mais aussi en leur disant comment ils peuvent s'améliorer.

\subsubsection{Les types de la retroaction}

Lyster et Ranta (1997: 37-66) cité par Hassan (2011: 63-65), explicitent les types de rétroaction:

- la correction explicite (en indiquant à l'étudiant qu'il y a eu une erreur, l'enseignant lui fournit la forme correcte).

- la reformulation (l'enseignant reformule la proposition de l'apprenant en éliminant l'erreur de façon implicite).

- la répétition de l'erreur, la demande de clarification (en se servant de phrases comme "Pardon?" ou "Je ne comprends pas", l'enseignant indique à l'apprenant que son message a été mal compris ou mal formulé).

- l'indice métalinguistique (à l'aide de commentaires mais sans lui donner la forme correcte, l'enseignant souligne un problème dans la déclaration de l'apprenant) .

- l'incitation (l'enseignant incite l'apprenant à trouver la forme correcte en commençant une phrase et en invitant l'apprenant à la compléter, ou bien en essayant de solliciter la forme correcte par le biais de questions, ou encore en demandant à l'apprenant de reformuler son énoncé).

De sa part, Ammar (2013: 3-6) propose que les types de rétroaction sont: la rétroaction corrective écrite directe inductive (explicite ou implicite) et la rétroaction corrective écrite déductive (métalinguistique ou incitation). Ces stratégiede rétroaction utilisées par les enseignants pour corriger les productions écrites de leurs étudiants.

\subsubsection{Les types d'erreurs et les techniques de la rétroaction}

Tout d'abord, l'erreur est un outil d'apprendre. Chacun de nous peut se demander s'il existe une relation entre le type d'erreurs et le type de rétroaction corrective fournie par l'enseignant. Il y a quatre principaux types d'erreurs (grammaticales, phonologiques, lexicales et usuelles non sollicitées) et de six techniques de rétroaction (correction explicite, reformulation, demande de clarification, rétroaction métalinguistique, incitation ourépétition).

Nishita (2004) a mis en évidence le fait que le type de rétroaction corrective utilisé dépend du type d'erreur. Par exemple, on utilise beaucoup la reformulation pour corrigerles erreurs syntaxiques et phonologiques, tandis que dans les études considérées, onpréconise l'incitation pour corriger les erreurs lexicales. (Hassan, 2011:70) 
L'apprentissage n'est en rien linéaire, il est spiralaire. Pour cela, l'erreur reflète également une prise de risque, un déséquilibre, une rupture nécessaire à l'apprentissage. Elle indique qu'une erreur a été commise sans toutefois fournir la forme requise Il existe plusieurs façons d'identifier les erreurs : souligner l'erreur, l'encercler, inscrire le nombre d'erreurs sur la ligne dans la marge, utiliser un code qui démontre où l'erreur se trouve et de quel type il s'agit. Les techniques de la retroaction dirigent l'attention de l'apprenant vers l'erreur et le laissent à lui-même pour résoudre et corriger le problème tout en étant plus ou moins guidé par le professeur. (Girard, 2015: 26)

D'après Sabry (2017: 64), il y a trois sous-catégories des erreurs relevées dans les productions écrites:

- Les erreurs de langue

Dans cette première catégorie, sont regroupées des erreurs de trois types:

- Le mot n'est pas identifié comme une entité de la chaine parlée (défaut de segmentation, oubli de mot. Ex.: « ils s'enalèrent ».

- La transcription du mot est incorrecte et ne correspond pas à sa réalisation orale: Ex.: « de parti d'ici ».

- Erreurs pouvant affecter la compréhension, portant sur les niveaux syntaxiques, lexicaux, et/ou langagières.

- Les erreurs portant sur l'orthographe grammaticale:

La deuxième catégorie regroupe les erreurs portant sur les accords erronés et la morphologie verbale. Beaucoup d'erreurs de ce type ont été relevées, sur la base d'une méconnaissance de certaines règles de morphologie verbale, d'accords en genre et en nombre erratiques, et de confusions sur les homophones.

- Les erreurs portant sur l'orthographe lexicale:

La troisième catégorie de cette typologie regroupe toutes les erreurs pour lesquelles le choix de graphie ne correspond pas à la norme, que ce choix de transcription soit adapté ou non à la réalisation phonétique du mot.

\subsubsection{Importance de l'étude des erreurs}

L'étude et l'analyse des erreurs représentent une grande importance dans le domaine de l'enseignement d'une langue et s'attachent à une démarche pédagogique proposée que Lamy (1976) a appelé "la pédagogie de la faute", consistant à éviter autant que possible la simple correction immédiate des erreurs produites par les apprenants et à mener un travail de (méta) réflexion plus approfondi. L'erreur est donc placée au centre de l'activité comme objet d'étude et de réflexion. Elle est importante: pour l'enseignant, pour l'apprenant et enfin pour le processus de l'apprentissage. 


\subsection{Pour l'enseignant}

En fait, l'analyse et le classement des erreurs peuvent être utiles aux enseignants parce que cela leur permettent de comprendre et préciser les problèmes rencontrés par les apprenants de F.L.E, ce qui les aident à connaitre leurs lacunes et explorer ainsi les démarches pédagogique. L'étude des erreurs des apprenants permet aussi à l'enseignant de différencier sa pédagogie et ses méthodes d'enseignement et évaluer sa performance.

\subsection{Pour l'apprenant}

En ce qui concerne les apprenants, l'étude des erreurs est aussi important pour eux, il leur permet de comprendre pourquoi il s'est trompé. Aussi, il lui donne l'occasion de corriger ses erreurs lui-même, il découvre donc son propre fonctionnement, ce qui l'encourage à plus d'autonomie.

\subsection{Pour le processus de l'apprentissage}

L'objectif général de l'étude des erreurs est le développement du processus de l'enseignement/apprentissage. Elle permet de mieux comprendre les processus d'apprentissage d'une langue étrangère et à découvrir la nature de ces processus au niveau psychologique.

Selon Besse \& Porquier (1991: 211), l'étude des erreurs apporte directement et indirectement une contribution importante à l'enseignement des langues dans deux domaines:

- pour améliorer les descriptions pédagogiques.

- pour modifier les attitudes et les pratiques d'enseignement et d'apprentissage.

Depuis longtemps, les erreurs en général et de l'écrit en particulier occupaient une place importante dans le domaine de l'enseignement/apprentissage du FLE. Les erreurs font ainsi partie de l'apprentissage, elles ne seront plus ressenties comme points de faiblesse mais un moyen d'apprendre et de progresser et un outil de formation plutôt qu'une production à corriger.

\section{Résultats de la recherche}

L'hypothèse de cette recherche réside qu'Il y a des différences significatives statistiquement au niveau $\mathrm{P}(\leq 0,05)$ entre les moyennes des notes des étudiants de l'échantillon de la recherche aux pré/post tests des compétences langagières pragmatiques en FLE en faveur du post test.

Pour vérifier cette hypothèse, le chercheur a utilisé «T» Test pour démontrer la signification statistique des différences entre les moyennes des notes des étudiants du groupe expérimental de la recherche. Il a également calculé la moyenne, l'écart type, le degré de la liberté, et la valeur de «T » au test des compétences langagières pragmatiques en FLE avant et après l'application le programme proposé basé sur la rétroaction. Le tableau N. ${ }^{\circ}$ (3) indique ces résultats. 
Tableau $\mathbf{N}^{\circ}(3)$

Test « $t$ » de la signification des différences entre les moyennes de notes des étudiants du groupe expérimental de la recherche aux pré/post-tests des compétences langagières pragmatiques en FLE

\begin{tabular}{|c|c|c|c|c|c|c|c|c|}
\hline $\mathbf{N}^{0}$ & Cométences & Application & $\mathbf{N}$ & M & E.T & D.L & $\mathbf{T}$ & $\mathbf{S}$ \\
\hline \multirow[t]{2}{*}{1.} & \multirow{2}{*}{ Pragmalinguistique } & \begin{tabular}{|c|} 
Pré \\
\end{tabular} & 30 & 6.1667 & 1.28877 & \multirow{2}{*}{29} & \multirow{2}{*}{9.724} & \multirow{2}{*}{0.001} \\
\hline & & Post & 30 & 8.6667 & 1.18419 & & & \\
\hline \multirow[t]{2}{*}{2.} & \multirow{2}{*}{ Discursive } & Pré & 30 & 4.2333 & 1.07265 & \multirow{2}{*}{29} & \multirow{2}{*}{10.346} & \multirow{2}{*}{0.001} \\
\hline & & Post & 30 & 7.7667 & 1.63335 & & & \\
\hline \multirow[t]{2}{*}{3.} & \multirow{2}{*}{ Informative } & Pré & 30 & 5.5000 & 1.16708 & \multirow{2}{*}{29} & \multirow{2}{*}{13.429} & \multirow{2}{*}{0.001} \\
\hline & & Post & 30 & 9.0667 & 1.08066 & & & \\
\hline \multirow[t]{2}{*}{4.} & \multirow{2}{*}{ Interaction sociale } & Pré & 30 & 2.4667 & .81931 & \multirow{2}{*}{29} & \multirow{2}{*}{8.762} & \multirow{2}{*}{0.001} \\
\hline & & Post & 30 & 4.2333 & .81720 & & & \\
\hline \multirow{2}{*}{\multicolumn{2}{|c|}{ Total }} & Pré & 30 & 18.3667 & 2.55266 & \multirow{2}{*}{29} & \multirow{2}{*}{28.441} & \multirow{2}{*}{0.001} \\
\hline & & Post & 30 & 29.7333 & 2.69013 & & & \\
\hline
\end{tabular}

\section{Légende:}

$\mathrm{N}$ : Nombre d'étudiants(es)

M.: Moyenne des Notes

E.T: Écart type

D.L: Degré de liberté

$\mathrm{T}$ : Valeur de « T »

S: Signification

Le tableau ci-dessus démontre qu'il existe une différence statistiquement significative au niveau de $P(\leq 0.01)$ entre les moyennes des notes des étudiants de l'échantillon de la recherche au pré/post test des compétences langagières pragmatiques en FLE en faveur du post test. Cette valeur met l'accent sur la progression dûe à l'utilisation du programme proposé basé sur la rétroaction et son impact observable sur le développement des compétences langagières pragmatiques en FLE. Donc, cette hypothése a été vérifiée.

Calul de l'effet du programme proposé basé sur la rétroaction sur les compétences langagières pragmatiques en FLE

Dans les lignes suivantes, on va présenter le calcul de l'effet du programme proposé basé sur la rétroaction comme suit:

Pour connaître la taille de l'effet de la variable indépendante sur la variable dépendante, le chercheur a calculé la valeur de «t», la valeur de Carré d'état en utilisant la formule suivante: $\left(\eta^{2}\right)$

Légende:

$$
\eta^{2}=\frac{t^{2}}{t^{2}+d l}
$$


ๆ2: Carré d'Eta. $\quad \mathrm{t}^{2}$ : Le Carré de la valeur de Test «t»

dl: degré de liberté

D'après Mansour (1997: 57-59) (Traduction de l'arabe), indique que les critères de la mesure de l'effet en termes du carré du « $\eta 2 »$ sont : 0.01; la petite mesure de l'effet, 0.06; la moyenne mesure de l'effet, plus de 0.15 , la grande mesure de l'effet. Le tableau ci-dessous indique la taille de l'effet du programme proposé basé sur la rétroaction sur le développement des compétences langagières pragmatiques en FLE.

Tableau $N^{\circ}(4)$

Taille de l'effet du programme proposé sur le développement des compétences langagières pragmatiques en FLE

\begin{tabular}{||c|c|c|c|c|}
\hline $\mathbf{N}^{\mathbf{0}}$ & Cométences & $\mathbf{T}$ & $\boldsymbol{\eta} \mathbf{2}$ & T.E \\
\hline 1. & Pragmalinguistique & 9.724 & 0.77 & élevé \\
\hline 2. & Discursive & 10.346 & 0.79 & élevé \\
\hline 3. & Informative & 13.429 & 0.86 & élevé \\
\hline 4. & Interaction sociale & 8.762 & 0.73 & élevé \\
\hline & Total & 28.441 & 0.97 & élevé \\
\hline
\end{tabular}

\section{Légende:}

T. : Valeur de « T » १2: Carré d'Eta

T.E.: Taille de l'effet

Le tableau ci-dessus démontre cette taille de l'effet qui était (0.97), donc, elle est significative et élévée. On trouve que (97\%) des changements qui se passent à la variable dépendante (les compétences langagières pragmatiques en FLE) reviennent à la variable indépendante (Le programme basé sur la rétroaction). Alors, ce programme proposé a un grand effet, donc, il efficace, parce que on peut mesurer l'impact d'un programme d'après son effet. 


\section{Discussion des résultats de la recherche}

À la lumière des résultats de la présente recherche, on trouve qu'il existe un développement observable dans la performance des étudiants de l'échantillon, les étudiants de la deuxième année au cycle secondaire concernant les compétences langagières pragmatiques en FLE aux pré/post tests en faveur du post test. Cela démontre l'effet de l'emploi du programme basé sur la rétroaction.

\section{Ce résultat peut être interprété comme suit:}

- Le programme d'entrainement a créé une atmosphère qui favorise l'acquisition des compétences langagières pragmatiques en FLE.

- Le programme basé sur les stratégiede la rétroaction a aidé les apprenants à utiliser la langue française dans la vie courante.

- Les interactions sociales faites entre les apprenants au sein des groupes leur ont donné l'occasion de dialoguer, de collaborer, d'exploiter un savoir- faire et de varier des acquis communicatifs et l'aspect pragmatique de la production écrite.

\section{Recommandations de la recherche}

D'après les résultats de cette recherche, le chercheur présente les recommandations suivantes:

- Développer les programmes proosés à la lueur des tendances modernes de l'enseignement et les principes du Cadre.

- Attirer l'attention des enseignants à se concentrer sur les compétences commuincatives (linguistiques, sociolinguistique et pragmatique).

- Motiver les étudiants à communiquer en langue étrangère à travers des situations de la vie quotidienne et mobiliser leurs capacités à s'exprimer entre eux dans des phrases simples et correctes.

\section{Suggestions de la recherche}

Cette recherche propose d'autres recherches telles que:

- Réappliquer les stratégiede la rétroaction sur d'autres cycles scolaires.

- Élaborer un programme basé les stratégie de la rétroaction pour developer les compétences langagières et professionnelles chez les enseignants en service.

- Utilisation de la rétroaction pour développer les compétences technologiques.

\section{Bibliographie}

Alrabdi, E. (2011). Quelle méthode faut-il adopter pour l'enseignement/apprentissage de l'oral?. Université du Yarmouk. Département de langues modernes. Vol. 23 15-34. Tiré de revistas.ucm.es/index.php/DIDA/article/download./36308/35153. 
Ammar, A. (2013). La rétroaction corrective écrite dans l'enseignement du français au Québec: Effets du type d'erreurs du profil de l'apprenant, $\mathrm{du}$ contexte d'apprentissage et de l'ordre d'enseignement. Etablissement gestionnaire de la subvention. Université de Montréal.

Asselin, C. (2006). Les rétroactions correctives à l'oral: que croient faire les enseignants et qu'en pensent les apprenants? Université du Québec à Montréal.

Awad, R. (2009). The effect of using a reflective model of teaching on developing second year English majors, self-assessment, self-steam and teaching skills. Minia university, Faculty of Education.

Awad, H. (2013). Utilisation de la grammaire récit pour développer les compétences de l'écriture chez les élèves du cycle préparatoire aux écoles francophones. Faculté de pédagogie, Université d'Ain Chams.

Brookhart, S. (2010). La rétroaction efficace. Des stratégiepour soutenir les élèves dans leur apprentissage. Adaptation : Léo-James Lévesque. Montréal : Chenelière Éducation.

Conseil de l'Europe (2001). Cadre européen commun de référence pour les langues: Apprendre, Enseigner, Évaluer. Paris: Didier.

Dalmasso, N. (2009). Etude du lien entre la compétence pragmatique et la compétence grammaticale au près d'apprentissage allophones de français L2. Université du Québec à Montréal.

Debanc, C. (1991). Comment les maîtres évaluent-ils les écrits de leurs élèves en classe?. Collections Rencontres Pédagogies, INRP- Hachette Éducation.

Desmson, F. (2005). Enseigner le FLE (français langue étrangère). Pratiques de class. Belin: Paris Cedeux.

Doré, Z. (1981). Influence relative de modèles de rétroaction dans l'apprentissage chez les enfants déficients mentaux moyens. Thèse de Doctorat. Université de Montréal.

El Shirbini, I. (2015). The effectiveness of some feedback stratégiein developing the EFL writing skills of secondary stage students and their attitudes towards writing. Mansoura university: Faculty of Education.

Fabienne, B. (2004). Mise au point d'un outil d'évaluation des capacités langagières pour une population d'enfants dysphasiques phonologicosyntaxiques et lexico-syntaxiques. Université libre de Bruxelles. Faculté de psychologie.

Fayol, M. (1996). La production du langage écrit. In J. David \& S.

Fayol, M. (1997). Des idées au texte. Paris: Presses universitaires de France. 
Girad, A. (2015). L'évaluation formative à l'école estonienne: les opnions des enseignants d'écoles élémentaires et des professeurs de FLE. Université de Tartu

Hassan, R. (2011). Les croyances des enseignants et des apprenants adultes quant à la rétroaction corrective à l'oral et la pratique réelle en classe de français langue étrangère en Egypte. Thése de doctorat. Faculté des études supérieures. Université de Montréal.

Hattie, J. (2007). The power of feedback. Review of Educational research, 77 (1), 81-112.

Kasper, G. et Rose, K. R. (2002). Pragmatic development in second language. University of Michigien.

Lyster, R. et Ranta, L. (1997). Corrective feedback and learning uptake: negotiation of form communicative classrooms. Studies in second language acquisition, 19, 37-66.

Mahmoud, A. (2003). The relative effectiveness of pragmatic approach on improving the comprehension and translation skills of students in the advanced-Level program at private school in Amman. Unpublished Thesis Ph.D. Amman Arab University Jordan.

Maraheel, W. (2004). The effect of using prgmatic competence test on the ninth Graders' proficiency of the major four skills in Nablus city, at an Najah national university, Nablus Palestine.

Martinot, D. (2006). Connaissance de soi, estime de soi et motivation scolaire. Paris: presse universitaire de France.

Mohamed, M. (2014). The effect of using some self-assessment techniques on developing some English writing skills among secondary stage students. Faculty of Education. Minoufiya University.

Noyon, Ch. (2011). Les interactions écrites en ligne en classe de français langue étrangère: élaboration d'un dispositif, analyse des discours produits et analyse des représentations. Thèse de Doctorat. Université Catholique de Louvain: Faculté de Philosophie, Arts et lettres.

Padru, M. (2015).la rétroaction corrective écrite en francisation au Quebec: les techniques, les types d'erreurs et les compétences des apprenants à l'écrit. Université de Montréal

Remue, E. (2004). Trois outils pour développer l'estime de soi en classe. Haute école de Léonard de Vinci, Louvain. la Neuve.

Sabry, A. (2017).Utilisation des ateliers de négociation graphiques pour remédier aux erreurs orthographiques chez les étudiants de la Faculté de Pédagogie - Section de Français. Faculté de pédagogie, Université de Mansourah 
Salama, S. (2010). The effects of using feedback techniques and error correction on EFL secondary school first year students' paragraph writing. Beni-Sueif University. Faculty of Education.

Tognet, C. (2014). Adaptation des deux épreuves de conversation fictive du test des habiletés langagières de B. Shalman. pour les enfants de 8 à 12 ans. Université Bordeaux Seglen.

Viljamaa, H. (2012). La compétence pragmatique en FLE: Emploi des requêtes et des remerciements chez les étudiants Finlandais. Université de Tuku: Institut de langues et de traduction. 